\title{
Prevalence of Allergic Rhinitis in China
}

\author{
Yuan Zhang, ${ }^{1,2}$ Luo Zhang ${ }^{1,2 *}$ \\ 'Department of Otolaryngology, Head and Neck Surgery, Beijing Tongren Hospital, Capital Medical University, Beijing 100730, PR China \\ ${ }^{2}$ Key Laboratory of Otolaryngology, Head and Neck Surgery (Ministry of Education of China), Beijing Institute of Otorhinolaryngology, Beijing 100005, PR China
}

This is an Open Access article distributed under the terms of the Creative Commons Attribution Non-Commercial License (http://creativecommons.org/licenses/by-nc/3.0/) which permits unrestricted non-commercial use, distribution, and reproduction in any medium, provided the original work is properly cited.

Allergic rhinitis (AR) is a serious systemic allergic disease, which together with comorbid asthma causes major illness and disability worldwide. Recent epidemiological studies have revealed wide variations in the increasing prevalence of AR and allergies globally, including in China. Despite a markedly higher population than western countries, and a landmass close to Europe in area, little epidemiological data is available on AR in China. Thus, the present study reviewed the prevalence, comorbid allergic diseases, trends and pattern of sensitizing allergens in adults and children suffering from AR in China. Available data indicated that despite variations in the prevalence of AR in different regions of the country, the prevalence of AR has increased in both adults and children over the last 2 decades. Similarly, there has been an increase in a "western"-type lifestyle, industrialization and air pollution over this period, which may have contributed to the increased prevalence of AR observed in China.

Key Words: Allergic rhinitis; prevalence; China; asthma; allergen; epidemiology; adult; children

\section{INTRODUCTION}

Allergic rhinitis (AR) is an inflammatory disease of the nasal mucosa, induced by an immunoglobulin E (IgE)-mediated reaction in allergen-sensitized subjects. AR is characterized by sneezing, rhinorrhea, nasal congestion and nasal pruritus, which are often accompanied by ocular pruritus, redness and/or lacrimation in $60 \%-70 \%$ of patients. ${ }^{1,2}$ Although not life threatening, the symptoms of AR are frequently bothersome, adversely affect work and quality of life, and impose a significant burden on both the individual and society. ${ }^{1-4}$ Symptoms can lead to both physical and mental complications, with sleep-disordered breathing in childhood and adolescence being associated with decreased learning performance and increased behavior and attention disorders. ${ }^{5-9}$ Moreover, AR is commonly comorbid with asthma, suggesting that these conditions are closely related and has led to the concept of 'one airway, one disease. ${ }^{10}$ Indeed, studies have suggested that $\sim 20 \%-50 \%$ of patients with allergic rhinitis have clinical asthma, whereas $>80 \%$ of patients with allergic asthma having concomitant rhinitis symptoms. ${ }^{11-13}$

Epidemiological studies have revealed that the prevalence of AR has increased progressively in more-developed countries, and currently affects up to $40 \%$ of the population worldwide ${ }^{14,15}$; with $23 \%-30 \%$ of the population affected in Europe $e^{16,17}$ and $12 \%$ $30 \%$ of individuals affected in the United States. ${ }^{18}$ Although the prevalence and possible factors responsible for the etiology of
AR have been well documented in many developed countries, there is comparatively little information available in developing countries. ${ }^{19}$ In this regard, the People's Republic of China is one of the largest countries in the world with a population of around 1.3 billion citizens and rapidly expanding industrialization. It is divided into 34 administrative divisions, including 23 provinces, 5 autonomous regions, 4 municipalities directly under the Central Government, and 2 Special Administrative Regions (Hong Kong and Macao). Moreover, it is traditionally divided into seven divisions, including North, Northeast, East, South, Northwest, Central and Southwest China, which cover an area of around 9.6 million square kilometers-approximately the size of Europe - and lies within several climate zones. The present article reviews the prevalence, incidence of comorbid allergic diseases, and trends and patterns of sensitizing allergens of AR in adults and children in China. The process of selecting articles to include in the present review was based on review of pre-existing published studies. A literature search was conducted in February 2013 for all relevant articles using PubMed. Cita-

Correspondence to: Luo Zhang, Professor, MD, Beijing Institute of Otolaryngology, No. 17, HouGouHuTong, DongCheng District, Beijing, 100005, China.

Tel: +8610-65141136; Fax: +8610-85115988; E-mail: dr.luozhang@gmail.com Received: April 26, 2013; Revised: July 19, 2013; Accepted: August 9, 2013

- There are no financial or other issues that might lead to conflict of interest. 
tions for articles reporting the prevalence of AR in China were identified using allergic rhinitis as the primary search terms in association with China, prevalence/incidence, comorbid allergic disease, asthma, and sensitizing allergens, as the secondary search terms.

\section{The prevalence of allergic rhinitis in adults}

Few studies have reported the prevalence of AR in adults in China (Figure). One nationwide population-based study assessed self-reported AR using validated questionnaire-based telephone interviews in over 38,000 subjects in 11 major cities across China from September 2004 to May $2005 .{ }^{20}$ The authors demonstrated that age- and gender-adjusted prevalence of AR was highly variable, ranging from $8.7 \%$ in Beijing to $24.1 \%$ in Urumqi. The prevalences of AR in the other regions investigated were $11.2 \%, 16.1 \%, 14.1 \%, 8.9 \%, 13.3 \%, 13.6 \%, 15.7 \%, 19.3 \%$, and $9.1 \%$ in Changchun, Changsha, Guangzhou, Hangzhou, Nanjing, Shanghai, Shenyang, Wuhan and Xi'An, respectively. Importantly, this study further showed that, of the subjects with self-reported AR, $74.4 \%$ were diagnosed as intermittent AR patients and the remaining $25.6 \%$ as persistent AR patients based on the new Allergic Rhinitis and its Impact on Asthma (ARIA) classification. ${ }^{21}$ More recently, Shen and colleagues ${ }^{22}$ investigated the epidemiological features of self-reported AR in 4 major cities (Chengdu, Chongqing, Nanning and Urumqi) in western China employing a cross-sectional, population-based study using a validated questionnaire and multistage, stratified cluster sampling from January to December 2008. The overall prevalence of AR was found to be $34.3 \%$, with prevalence rates of $34.3 \%$ in Chengdu, 32.3\% in Chongqing, 30.3\% in Nanning, and 37.9\% in Urumqi. Similarly, in more recent studies, Wang and colleagues investigated the prevalence of AR in several regions of

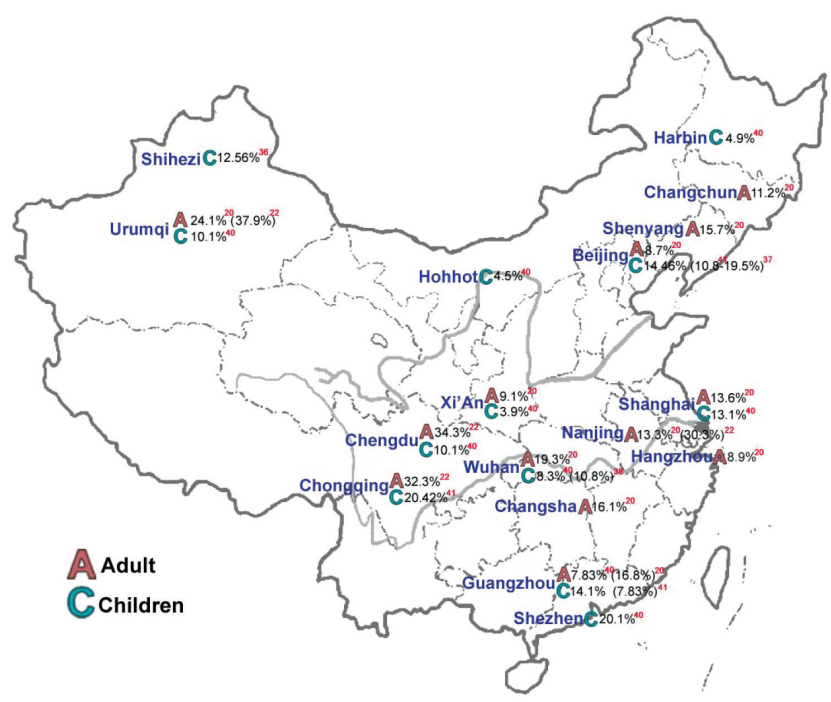

Figure. Reprehensive published prevalence of allergic rhinitis in adults and children in different cities in China. northern China (rural areas of Qingxian, Hebei; coastal fishing village of Bohai Bay, Huanghua; area of Wuling Mountain, Chengde; urban areas of Tianjin) and reported that the overall prevalence of AR was $\sim 9.2 \%{ }^{23,24}$ The authors further demonstrated that the risk of AR was associated with occupation, with farmers having a 2.32-fold increased risk. ${ }^{23}$ Comparison of AR prevalence in villagers in Hebei Province versus city dwellers in Tianjin indicated that although the prevalence rates $(9.2 \%$ and $9.0 \%$, respectively) were not significantly different, the prevalence of allergens in the two areas were significantly different. ${ }^{25}$ The incidence of AR caused by dog epithelium was higher in Hebei than Tianjin, whereas the reverse was the case for dustmite-induced AR.

\section{The prevalence of allergic rhinitis in children}

Compared to adults, more data are available from studies investigating the prevalence of AR in children in China (Figure), the majority of which have investigated the prevalence of AR in combination with asthma and eczema using the standardized and appropriately translated versions of the International Study of Asthma and Allergies in Childhood (ISAAC) protocols. ${ }^{10,26,27}$ Despite being approximately similar in size and having a population twice as large as Europe, only 2 centers in China have reported the prevalence of AR and/or asthma/eczema in children aged 6-7 years according to the ISAAC protocol, compared with 29 centers in Europe. Likewise, the prevalence of AR and or asthma/eczema in children aged 13-14 have been investigated in only 3 centers in China, compared with 46 centers in Europe. ${ }^{28}$

In an early, non-ISAAC design study, Leung and colleagues investigated the effects of allergen sensitization and a family history of atopy on asthma and allergic disease in 12-18 years old Chinese schoolchildren from San Bu in southern China and Hong Kong. ${ }^{29}$ The authors reported that the occurrence rates of rhinitis symptoms within the past 12 months in San $\mathrm{Bu}$ and Hong Kong were $3.7 \%$ and $29.8 \%$, respectively, while the rates of atopy confirmed by skin prick tests (SPT) were $49 \%$ and $57.7 \%$, respectively, in the 2 regions..$^{29}$ Similarly, 2 earlier ISAAC Phase I studies investigating 6-7 years old primary school children in Beijing $(\mathrm{n}=2,978)$, Urumqi $(\mathrm{n}=2,840)$ and Hong Kong $(\mathrm{n}=3,618)$ reported 12 month prevalence rates for nasal symptoms of $30.0 \%, 31.1 \%$, and $35.1 \%$, respectively. ${ }^{30,31}$ Wong et al..$^{32}$ recruited schoolchildren aged 9-11 years from Hong Kong $(n=3,110)$, Beijing $(n=4,227)$ and Guangzhou $(n=3,565)$ for studies using the ISAAC Phase II protocol, and reported that the prevalence rate of current rhinoconjunctivitis was significantly higher in Hong Kong (15\%) than in Beijing (6.7\%) or Guangzhou (7.4\%). The atopy rate was also higher in Hong Kong (41.2\%) than in Beijing (23.9\%) or Guangzhou (30.8\%). In a more recent study, schoolchildren aged 13-14 years from urban Beijing $(n=3,531)$ and rural areas around Beijing $(n=3,546)$ were assessed using the ISAAC Phase III protocol..$^{34}$ The incidence rates of current symptoms of rhinoconjunctivitis and atopy were significantly 
higher in urban children ( $10.3 \%$ and $37 \%$, respectively) compared with rural children ( $3.3 \%$ and $11 \%$, respectively; $P<0.0001$ for both comparisons) ${ }^{33}$ Similarly, Chiang and colleagues ${ }^{34}$ investigated the prevalence of AR in 10-15 years old schoolchildren in central Taiwan using the ISAAC Phase III study protocol. These authors reported the overall cumulative and 12 month prevalence of AR to be even higher in this cohort, at $43.0 \%$. Moreover, the authors concluded that boys had significantly higher prevalences of wheezing and rhinitis than girls, and that younger children tended to have a higher prevalence of the disorders than older children. ${ }^{34}$ This observation confirmed the age- and gender-dependent differences in AR prevalence shown by Kao and colleagues ${ }^{35}$ in an earlier non-ISAAC designed study employing a one-stage stratified cluster random sampling technique. These authors investigated the prevalence of AR in 2 groups of school children in Taiwan and demonstrated that children aged 6-8 years had significantly higher prevalence rates of diagnosed AR than those aged 13-15 (29.8 vs $18.3 \%$ ), and the 6-8-year-old male group had significantly higher proportions of AR symptoms than females of similar age ${ }^{36}$ Likewise, one recent non-ISAAC questionnaire-based study has reported the prevalence rate of AR in children aged 9 to 10 years in Shihezi city of Xinjiang province located in Northwest China to be $12.56 \%{ }^{36}$ Moreover, the authors showed that $56 \%$ of children with AR had intermittent AR, and the remaining $44 \%$ had persistent AR with mostly mild symptoms based on the ARIA classification. ${ }^{21}$

Several studies have investigated the prevalence of AR in preschool children aged 6 years and less. ${ }^{37,38}$ In one recent study, Zhang and colleagues ${ }^{37}$ surveyed 4,075 children aged 3 to 5 years in urban and suburban areas of Beijing; the adjusted prevalence rate of $19.5 \%$ for clinical AR was significantly higher in children from urban areas, compared to $10.8 \%(P<0.01)$ in children from suburban areas. Moreover, $67.1 \%$ of the children with AR were found to be suffering from intermittent $\mathrm{AR}$, and $32.9 \%$ from persistent AR, according to the ARIA classification ${ }^{21}$; with $41.5 \%$ of the patients showing moderate/severe symptoms. ${ }^{37}$ In another recent study, Kong and colleagues ${ }^{38}$ surveyed 1,211 children aged 3-6 years from 16 kindergartens in Wuhan using author-generated questionnaires followed up by telephone interviews and skin prick testing. The authors reported the adjusted current prevalence of AR to be $10.8 \%$ using the diagnostic criteria based on the presence of nasal symptoms and positive skin prick test (SPT). Moreover, the prevalence of AR was higher in males than females $(13.0$ vs $7.7 \% ; P<0.05)$ and the percentage of children sensitive to both inhalant and food allergens was $27.4 \%{ }^{38}$ One study investigated the relativity of AR rhinitis and recurring respiratory infection in preschool children in Shenzhen, Guangdong province in southern China. They demonstrated that, while the prevalence rates of AR and recurring respiratory infection were $20.1 \%$ and $13.7 \%$, respectively, the prevalence rate of $18.0 \%$ for AR combined with recurring respirato- ry infection was significantly higher than the prevalence rate of $7.8 \%(P<0.05)$ for cases with non-AR combined recurring respiratory infection. ${ }^{39}$

To date, only one nationwide survey of the prevalence of AR in children in China has been reported. ${ }^{40} \mathrm{~A}$ total of 23,791 children aged 6-13 years in 8 metropolitan capital cities (Shanghai, Guangzhou, Xi'an, Wuhan, Harbin, Chengdu, Hohhot and Urumqi) of provinces in 4 regions were surveyed during November and December 2005, using a cluster-stratified sampling method. Three key questions from the standard ISAAC questionnaire were used to examine the prevalence of current allergic rhinitis and allow for comparison with results in the literature. The study demonstrated that the mean prevalence of childhood AR in the 8 cities was $9.8 \%$, and ranged from $3.9 \%$ in Xi'an to $16.8 \%$ in Guangzhou, with males showing a higher AR prevalence than females in all cities. Moreover, the prevalence of childhood allergies was associated with factors related to lifestyle, mental health and socio-economic status, which were distributed unevenly across cities and disproportionately affected the local prevalence. ${ }^{40} \mathrm{~A}$ smaller cross-sectional survey by Zhao and colleagues ${ }^{41}$ investigated the self-reported prevalence of childhood AR in three major cities in China (Beijing, Chongqing, and Guangzhou) using the ISAAC questionnaire. A total of 24,290 children aged 0-14 years were interviewed using a multi-stage sampling method; the self-reported prevalences of AR were $14.46 \%, 20.42 \%$, and $7.83 \%$ in Beijing, Chongqing and Guangzhou, respectively. Furthermore, the prevalence of AR was lower in children younger than 2 years and significantly higher in males than females in each city. ${ }^{41}$

\section{The prevalence of allergic rhinitis and comorbid allergic diseases}

Atopic dermatitis (AD) is the cutaneous precursor of an evolving systemic disorder, the "atopic march", which progresses from $\mathrm{AD}$ to $\mathrm{AR}$ and asthma. ${ }^{42,43}$ There is a close relationship between AR and asthma, which has led to the concept of "the united airway" / "one-airway, one disease,"10 with evidence suggesting that AR is an independent risk factor for the development of asthma. ${ }^{21,44}$ Using a 2-stage, clustered, stratified random sample study design, Zhang and colleagues ${ }^{38}$ surveyed 4,075 children aged 3, 4 , and 5 years in urban and suburban areas in Beijing and estimated that $13.3 \%$ and $18.1 \%$ of children with clinical AR also suffered from physician-diagnosed comorbid asthma and eczema, respectively. Furthermore, there was a close association between AR and asthma in children with a history of asthma $(P<0.01, \mathrm{OR}=4.88)$ or eczema $(P<0.01, \mathrm{OR}=1.49)$, suggesting that both asthma and eczema were likely to be significant concomitant risk factors for AR. ${ }^{37}$ Another recent random, multistage, cluster sampling study by Wang and colleagues ${ }^{23}$ analyzed the correlation between $\mathrm{AR}$ and bronchial asthma or $\mathrm{AD}$ in a cohort of 1,524 individuals from several regions of northern China. This study reported that in patients with AR, the preva- 
lences of bronchial asthma and $\mathrm{AD}$ were significantly higher, at $30.9 \%$ and $29.5 \%$, respectively, compared with those of bronchial asthma and $\mathrm{AD}$ of $2.9 \%$ and $3.7 \%$ ( $P<0.01$ for both), respectively, in subjects without AR. Additionally, the risk of subjects with asthma suffering from AR was 8.619-fold higher than that of subjects without bronchial asthma, whereas the risk of subjects with $\mathrm{AD}$ suffering from $\mathrm{AR}$ was 1.817-fold higher than that of subjects without $\mathrm{AD}^{23}$ Yin and colleagues ${ }^{45}$ investigated the relationship between AR and allergic asthma in 1,120 patients with autumnal pollinosis in Beijing, and reported that $1,096(97.9 \%)$ patients had AR, whereas only $10(0.9 \%)$ had allergic asthma. Furthermore, of the 1,096 patients with AR, 585 (53.4\%) suffered from seasonal asthma, suggesting that the prevalence of autumnal comorbid asthma and AR in Beijing may be relatively high. In contrast, a study of 2,205 elementary school children with AR in Xinjiang province in northwest China reported that of the 277 (12.56\%) children found to be suffering from AR, 15 (5.4\%) were also suffering from comorbid bronchial asthma. ${ }^{36}$

\section{Trends in the prevalence of allergic rhinitis}

The worldwide incidence and prevalence of AR have been increasing over the last 3 decades ${ }^{28,46-49}$ in almost all westernized countries. A recent study by de Marco and colleagues ${ }^{50}$ of the trends in the prevalence of asthma and AR in Italy between 1991 and 2010 reported that the prevalence of AR has increased continuously in Italy during this time, affecting more than one in 4 young adults by the end of 2010 . Several studies have documented the trends in AR prevalence in China. Studies in adults have demonstrated that the prevalence of AR in Nanjing and Urumqi have increased from $13.3 \%$ and $24.1 \%$ in $2004-2005^{20}$ and to $30.3 \%$ and $37.9 \%$ in $2008,{ }^{22}$ respectively, indicative of a dramatic increase in AR in Chinese adults. Similarly, ISAAC studies have indicated that the prevalence of physician-diagnosed hay fever and AR in Chinese school children aged 13-14 years increased significantly from $2.9 \%$ and $17.4 \%$ (noted in a Phase I study conducted in 1994-1995) and from $4.1 \%$ and $22.7 \%$, respectively (noted in a Phase III study conducted in 2001) (both $P<0.05) .{ }^{51}$ Likewise, an epidemiological survey of school children aged 6-7 years in Hong Kong from 1995 to 2001 reported that the prevalences of life-time rhinitis ( $42.4 \%$ vs $38.9 \%, P<$ 0.01 ), current rhinitis ( $37.4 \%$ vs $35.1 \%, P<0.03$ ), and current rhinoconjunctivitis ( 17.2 vs $13.6 \%, P<0.01$ ) have increased significantly over time. ${ }^{31}$ Similarly, 3 large sample epidemiological surveys of the prevalence of AR in schoolchildren in Taichung, central Taiwan, conducted in 1987, 1994, and 2002 have shown a progressive increase in the prevalence of AR from $5.1 \%$ in 1987, to $12.46 \%$ in 1987 , and finally $27.59 \%$ in $2002 .{ }^{52}$

\section{Pattern of sensitization to inhaled allergens in allergic rhinitis}

Several studies have investigated the prevalence and type of inhaled sensitizing allergens involved in the manifestation of $\mathrm{AR}$ in various cities and regions in China (Table 1), the majority of which were performed in a single city. Dust mites were reported as the most common allergen in many regions, whereas Chenopodium and Lupulus (instead of dust mites) were the main aeroallergens in northwestern China. Compared with adults, studies of the sensitizing inhaled allergens in children with AR suggested that a wider spectrum and Alternaria tenuis as well as mixed fungi were more common in children than adults.

A representative study by Yang and colleagues ${ }^{53}$ investigated

Table 1. Reprehensive studies documenting spectrum of inhaled allergens of AR in China

\begin{tabular}{llll}
\hline Regions & \multicolumn{1}{c}{ Study subjects } & \multicolumn{1}{c}{ Main allergens } & Year \\
\hline Beijing & Adults and Children & Male: Derf, Der , mugwort and BlaterliaGermanica & $2011^{53}$ \\
Kunshan & Adults & Female: Derf, Der p, mugwort and Chenopodiumalbum & $2012^{54}$ \\
Shanghai & Adults & Dust mite and house dust mite & $2012^{55}$ \\
Changsha & Adults & Flour mite, house dust mite, mycetes Il and weed & $2011^{56}$ \\
Enshi & Adults & Mites & $2011^{57}$ \\
Jingmen & Adults & House dust mite, Derf, mugwort and ragweed & $2010^{58}$ \\
Beijing & Adults & Derf, Der $p$, flowers pollen and herbs & $2006^{59}$ \\
Changji & Adults & Chenopodium and lupulus & $2011^{61}$ \\
Anqing & Adults & Silk & $2001^{62}$ \\
Beijing & 3-5-year-old children & Alternariatenuis, Der fand Der $p$ & $2013^{37}$ \\
Beijing & 1-3-year-old children & Mixed fungal, dust mite and mugwort \\
Changsha & Children & Derfand Der $p$ & $2011^{65}$ \\
Wuhan & 3-6-year-old children & House dust mites and moulds & $2011^{66}$ \\
Shihezi & 9-10-year-old children & Mugwort and house dust mite & $2009^{38}$ \\
\hline 11 major cities & Adults & Pollen, animal dander and dust mites & $2009^{36}$ \\
\hline
\end{tabular}


the patterns of sensitization to the main inhaled allergens in AR patients nationwide. A total of 10,030 patients with AR were divided into eight groups according to gender and age, and the distributions of sensitizing allergens were analyzed using SPTs. The 4 most common allergens in male patients with AR were Dermatophagoides farina (Derf), Dermatophagoides ptoronyssinus (Der $p$ ) mugwort and Blaterlia Germanica, while Der $f$, Der $p$, mugwort and Chenopodium album were the most common in female patients. In all groups, the majority of patients were sensitized to 2 relevant allergens, followed by polysensitization to 3 positive allergens and a single positive allergen. Furthermore, the top 4 allergens in 3-17-year-old males sensitized to a single allergen were $\operatorname{Der} f$, Der $p$, Alternaria tenuis and mugwort. In all other age groups, the most common positive allergens were Derf, Der p, mugwort and Blaterlia Germanica. In contrast, the top 4 positive allergens in the 3-17-year-old female group sensitized to a single allergen were $\operatorname{Der} f$, Der $p$, mugwort and Alternaria tenuis, in the 18-39-year-old group were $\operatorname{Der} f$, Der $p$, mugwort and Dandelion, in the 40-59-year-old group were $\operatorname{Der} f$, $\operatorname{Der} p$ mugwort and mixed tree 1, and in the $\geq$ 60-year-old group were $\operatorname{Der} f$, $\operatorname{Der} p$, mixed animal hair and mugwort. Additionally, in 3-17-year-old patients sensitized to 2 allergens, the most common allergen combination was $\operatorname{Der} f$ and $\operatorname{Der} p$, while in patients polysensitized to 3 positive allergens, the most common allergen combination was $\operatorname{Der} f$, Der $p$ and Blaterlia Germanica, followed by Der f, Der $p$ and mixed animal hair. While data from several other studies conducted in different regions in China have also shown $\operatorname{Der} f$ and $\operatorname{Der} p$ to be the most common sensitizing allergens in adult patients with AR in those areas, ${ }^{54-60}$ other authors have reported discrepant findings. A study investigating sensitization to inhaled allergens in AR patients in Xinjiang, Northwest China, demonstrated that Chenopodium and Lupulus (the main aeroallergens in the area) were the primary sensitizing agents for AR, whereas the prevalence of AR due to dust mites, fungus and canine epidermis was relatively low. ${ }^{61}$ A population-based study examining the relationship between asthma, AR and skin test reactivity to aeroallergens in over 10,000 subjects in Anqing (a predominantly rural province in China) has reported that sensitization to silk was important in the etiology of AR. While sensitization to perennial aeroallergens was associated with asthma among families of asthmatic subjects, sensitization to silk was the strongest predictor of rhinitis in this population. ${ }^{62}$ Interestingly, one study reported that the prevalence of positive cockroach SPT was higher in southern than northern China, in adults than children, and in males than females. ${ }^{63}$ Moreover, a cross-sectional study of the association between asthma and rhinitis severity and degree of specific allergic sensitization to common aeroallergens in AR patients in 24 centers (covering mid-temperate, warm-temperate, subtropical and tropical zones) across China reported that while moderate-severe intermittent rhinitis was significantly associated with the skin wheal size and the level of sIgE to Artemisia vulgaris and Ambrosia artemisifolia, moderate-severe asthma was significantly associated with increasing wheal size and the sIgE response to Der $p$ and $\operatorname{Der} f^{64}$ A survey of 3-5-year-old preschool children with AR in urban and suburban areas of Beijing revealed that the most common inhalant allergens were Alternaria tenuis, followed by Der $f$ (39.4\%), and $\operatorname{Der} p(38.6 \%){ }^{37}$ Similarly, another study of 1-3-yearold children with AR in Beijing reported that the most common inhaled sensitizing aeroallergens in these children were mixed fungal (50.0\%), followed by dust mite $(21.0 \%)$ and mugwort (12.5\%) ${ }^{65}$ However, 2 studies conducted respectively in Changsha ${ }^{66}$ and Wuhan, ${ }^{38}$ cities in central China, reported that the most common sensitizing allergens in children with AR were house dust mites, thus presenting an allergen spectrum in accordance with that of adults. Similarly, a study by Han and colleagues ${ }^{36}$ in 9-10-year-old AR children in Shihezi, Xinjiang province in Northwest China, reported mugwort and house dust mite allergens to be the most common sensitizing agents for AR.

Recently, pollen-related AR has attracted more attention in China, as pollen was the most commonly reported allergen $(47.8 \%)$ in a study by Zhang and colleagues. ${ }^{20} \mathrm{~A}$ study conducted between November 2003 to October 2004 to investigate the general and seasonal distribution of airborne pollen and its relationship with pollinosis in 16 areas in 12 cities in Hubei province, central China ${ }^{67}$ identified 61 pollen genera within the 257,520 collected pollen samples. The peak airborne pollen distribution occurred in 2 seasons each year; spring (March and April) and autumn (August to October), and pollinosis corresponded to the peak pollen distribution. Similarly another study of airborne pollens performed in Beijing indicated that the summer-autumn pollen concentration peaked from August 20 to September 15, with the major pollens being Artemisia $L$, Chenopodium album and Humulus scandens. ${ }^{68}$ Moreover, there was a significant correlation between specific pollen concentration and the number of patients sensitized to that pollen, as well as between pollen concentration and the onset of symptoms during the summer-autumn pollen season. ${ }^{68}$

\section{Influence of air pollution on the prevalence of allergic rhinitis in China}

There is accumulating evidence that both genetic and environmental factors play important roles in the etiology of $A R$, and that even small increases in the daily concentrations of air pollutants can cause adverse health effects. ${ }^{69}$ Recent studies have shown an association between high levels of air pollution and an increased risk of allergic sensitization and prevalence of rhinitis. ${ }^{70,71}$ The increase in atmospheric pollutant $\left(\mathrm{NO}_{2}, \mathrm{SO}_{2}\right.$, $\mathrm{PM}_{10}$, etc.) levels could explain the current increase in the prevalence of $\mathrm{AR}^{72-74}$

China is undergoing urbanization and westernization at an unprecedented rate, and the rapid increase in motor vehicle ownership and use has been accompanied by a concomitant 
increase in traffic-related air pollution, which poses an increasingly serious threat to health in urban areas of China. This is exemplified by a recent ecological study, which investigated the impact of traffic control measures and weather conditions on air quality and asthma morbidity over a period of 41 days during the 2008 Summer Olympic Games in Beijing. ${ }^{75}$ The study demonstrated that compared with the pre-Olympic baseline, the Games were associated with a significant reduction in asthma visits (RR 0.58, 95\% CI: 0.52-0.65), thus providing support for the concept that reduction of air pollution by reducing motor vehicle traffic could lead to improved health. The effects of traffic-related particulate air pollutants on AR prevalence have been investigated by several studies. Zhang and colleagues ${ }^{76}$ assessed the potential effects of $\mathrm{PM}_{10}, \mathrm{SO}_{2}$ and $\mathrm{NO}_{2}$ on outpatient visits caused by AR in Beijing during the period 2009-2010, and found strong associations between the daily concentration of the 3 air pollutants and the daily number of outpatients for $\mathrm{AR}^{76}$ Similarly, another cross-sectional, population-based study conducted in 11 major cities in China reported that the adjusted self-reported prevalence of AR was positively correlated with the concentration of $\mathrm{SO}_{2}$, but not with other air pollution factors, including $\mathrm{NO}_{2}$ and $\mathrm{PM}_{10}$ or any meteorological factors, including annual average temperature, annual average relative humidity, annual hours of sunshine and annual precipitation. ${ }^{20}$ Likewise, studies of the relationship between traffic-related air pollution and AR in Taiwanese schoolchildren have shown associations between the prevalence of $\mathrm{AR}$ and levels of $\mathrm{SO}_{2}, \mathrm{NOx}$, $\mathrm{O}_{3}$, and $\mathrm{PM}_{10}{ }^{77,78}$ Moreover, a study conducted to determine the relationship between air pollution and the prevalence of allergic diseases in the city of Taichung and the rural town of ChuShan in Taiwan revealed that the prevalence AR is higher in Taichung than Chu-Shan, suggestive of a potential adverse healthrelated effect of higher levels of air pollutants in the urban location. ${ }^{79}$ In contrast to traffic-related air pollutants, a study of the correlation between the incidence of Asian dust storms in Taipei, Taiwan (1997-2001), and the daily clinic visits for AR showed that although the effects of dust storms on clinic visits for AR were prominent 2 days after the event (19\%), this association was not statistically significant. ${ }^{80}$

\section{Limitations of the epidemiology of allergic rhinitis in China}

Although many studies have investigated the prevalence of AR in children and adults in China, the majority included disease in children and demonstrated obvious regional differences. Those results suggest that industrialization as the gross output value of industry of the most developed cities such as Beijing and Shanghai is much higher than that of the least developed cities. However, we did not identify a strong correlation between the prevalence of AR and major socioeconomic indicators, which may be due to the limitations of the published studies. A major limitation of these studies is that it is difficult to compare the prevalence of AR between populations since uni- form methods were not used. In this sense, it is likely that the marked variation in the prevalence of AR and the comorbid allergic diseases documented in these studies was due at least in part to differences in the instruments employed to assess prevalence. Additionally, many studies evaluated the prevalence of AR based only on questionnaire surveys, telephone interviews and self-reported symptoms. In this regard, it is possible that patients with non-AR, such as vasomotor rhinitis, eosinophilic nonallergic rhinitis and infectious rhinitis caused by viruses, were included in this data, leading to an artificially higher prevalence, particularly in the absence of allergen-test related data. Moreover, for the majority of studies based on questionnaire surveys only, there may be an overestimation of up to $50 \%$ in the prevalence of AR. Indeed, a recent study by Zhang and colleagues ${ }^{37}$ demonstrated that the prevalence of AR assessed using only epidemiological criteria was 3.2 folds greater (48 vs $14.9 \%$ ) than that assessed using diagnostic criteria, such as a combination of a typical history of AR symptoms and allergy tests (e.g. SPT). In contrast, one study reported that the prevalence of AR was underestimated on the basis of a lower number of patients being treated for AR, primarily because a majority of patients with mild symptoms did not feel the need to see a physician and did not attend the clinic. ${ }^{81}$ This study emphasizes the importance of improving utilization of resident health services and related medical education. Zhang and colleagues ${ }^{82}$ investi- $^{-}$ gated the understanding among otolaryngologists of the perspectives on the diagnosis and management of AR in major cities in China, and reported that the majority of the otolaryngologists $(61 \% \pm 29 \%)$ diagnosed AR based on medical history and nasal examination, while only one third $(35 \% \pm 28 \%)$ used SPT or specific IgE serum tests. These findings suggest that the diagnosis and management of AR in China is sub-optimal and that otolaryngologists require continuous education in aspects of the diagnosis and management of $\mathrm{AR}^{82}$

\section{CONCLUSIONS}

This study has reviewed the data of published surveys of the prevalence of AR and the risk and associated factors in several regions of China. Despite the greater land area and population compared to many western countries, little information is available on the epidemiology of AR in China. Available data indicate that although there are variations in the prevalence of AR among regions, the prevalence of AR has generally increased in both adults and children over the last 2 decades. This is in agreement with the trends noted for AR prevalence in other developing countries. While differences in the type, amount and length of exposure to naturally occurring biological sensitizers among regions may explain the differences in the prevalence of $A R$, it is likely that a "western" type lifestyle in combination with industrialization and a substantial rise in levels of fossil fuel-derived air pollutants has contributed to the increased prevalence of $A R$ 
in many areas of China.

As a developing country, gaps with regard to the socioeconomic level and the degree of technological development remain between China and the more developed westernized countries, which can lead to difficulties in performing epidemiological studies of AR. Moreover, surveying techniques are not standardized, which makes it difficult to directly compare the epidemiology of AR among areas, sub-populations and age groups in China. Thus, future studies of the epidemiology of AR in children and adults in China should be performed at the national level, with a clinical diagnosis of AR confirmed by allergen-related examinations and employing a standardized methodology.

\section{ACKNOWLEDGMENTS}

This work was supported by grants from National Science Fund for Distinguished Young Scholars (81025007), National Natural Science Foundation of China (30973282 and 81100706), Beijing Natural Science Foundation (7131006), the Special Fund of Sanitation Elite Reconstruction of Beijing (2009-2-007), Ministry of Health Foundation (201202005), Beijing Science and Technology program (Z111107055311040, KZ201110025027 and Z121107009212032) and Beijing Nova Program (2010B022) to LZ and YZ.

\section{REFERENCES}

1. Canonica GW, Bousquet J, Mullol J, Scadding GK, Virchow JC. A survey of the burden of allergic rhinitis in Europe. Allergy 2007;62 Suppl 85:17-25.

2. Schatz M. A survey of the burden of allergic rhinitis in the USA. Allergy 2007;62 Suppl 85:9-16.

3. Schoenwetter WF, Dupclay L Jr, Appajosyula S, Botteman MF, Pashos CL. Economic impact and quality-of-life burden of allergic rhinitis. Curr Med Res Opin 2004;20:305-17.

4. Reed SD, Lee TA, McCrory DC. The economic burden of allergic rhinitis: a critical evaluation of the literature. Pharmacoeconomics 2004;22:345-61.

5. Nathan RA. The burden of allergic rhinitis. Allergy Asthma Proc 2007;28:3-9.

6. Blaiss MS. Pediatric allergic rhinitis: physical and mental complications. Allergy Asthma Proc 2008;29:1-6.

7. Lv X, Han D, Xi L, Zhang L. Psychological aspects of female patients with moderate-to-severe persistent allergic rhinitis. ORL J Otorhinolaryngol Relat Spec 2010;72:235-41.

8. Lv X, Xi L, Han D, Zhang L. Evaluation of the psychological status in seasonal allergic rhinitis patients. ORL J Otorhinolaryngol Relat Spec 2010;72:84-90.

9. Xi L, Zhang Y, Han D, Zhang L. Effect of asthma, aeroallergen category, and gender on the psychological status of patients with allergic rhinitis. J Investig Allergol Clin Immunol 2012;22:264-9.

10. Asher MI, Keil U, Anderson HR, Beasley R, Crane J, Martinez F, Mitchell EA, Pearce N, Sibbald B, Stewart AW, Strachan D, Weiland SK, Williams HC. International Study of Asthma and Allergies in
Childhood (ISAAC): rationale and methods. Eur Respir J 1995;8: 483-91.

11. Peroni DG, Piacentini GL, Alfonsi L, Zerman L, Di Blasi P, Visona' G, Nottegar F, Boner AL. Rhinitis in pre-school children: prevalence, association with allergic diseases and risk factors. Clin Exp Allergy 2003;33:1349-54.

12. Chen J, Kong W, Xiang J, Shu H, Shi Q, Tan H, Zhou Y. Age features of the allergens in allergic rhinitis patients of different age in Hubei area. Lin Chung Er Bi Yan Hou Tou Jing Wai Ke Za Zhi 2008;22:6835,694 .

13. Strachan D, Sibbald B, Weiland S, Aït-Khaled N, Anabwani G, Anderson HR, Asher MI, Beasley R, Björkstén B, Burr M, Clayton T, Crane J, Ellwood P, Keil U, Lai C, Mallol J, Martinez F, Mitchell E, Montefort S, Pearce N, Robertson C, Shah J, Stewart A, von Mutius E, Williams H. Worldwide variations in prevalence of symptoms of allergic rhinoconjunctivitis in children: the International Study of Asthma and Allergies in Childhood (ISAAC). Pediatr Allergy Immunol 1997;8:161-76.

14. Bousquet J, Khaltaev N, Cruz AA, Denburg J, Fokkens WJ, Togias A, Zuberbier T, Baena-Cagnani CE, Canonica GW, van Weel C, Agache I, Aït-Khaled N, Bachert C, Blaiss MS, Bonini S, Boulet LP, Bousquet PJ, Camargos P, Carlsen KH, Chen Y, Custovic A, Dahl R, Demoly P, Douagui H, Durham SR, van Wijk RG, Kalayci O, Kaliner MA, Kim YY, Kowalski ML, Kuna P, Le LT, Lemiere C, Li J, Lockey RF, MavaleManuel S, Meltzer EO, Mohammad Y, Mullol J, Naclerio R, O'Hehir RE, Ohta K, Ouedraogo S, Palkonen S, Papadopoulos N, Passalacqua G, Pawankar R, Popov TA, Rabe KF, Rosado-Pinto J, Scadding GK, Simons FE, Toskala E, Valovirta E, van Cauwenberge P, Wang DY, Wickman M, Yawn BP, Yorgancioglu A, Yusuf OM, Zar H, Annesi-Maesano I, Bateman ED, Ben Kheder A, Boakye DA, Bouchard J, Burney P, Busse WW, Chan-Yeung M, Chavannes NH, Chuchalin A, Dolen WK, Emuzyte R, Grouse L, Humbert M, Jackson C, Johnston SL, Keith PK, Kemp JP, Klossek JM, Larenas-Linnemann D, Lipworth B, Malo JL, Marshall GD, Naspitz C, Nekam K, Niggemann B, Nizankowska-Mogilnicka E, Okamoto Y, Orru MP, Potter P, Price D, Stoloff SW, Vandenplas O, Viegi G, Williams D; World Health Organization; GA(2)LEN; AllerGen. Allergic Rhinitis and its Impact on Asthma (ARIA) 2008 update (in collaboration with the World Health Organization, GA(2)LEN and AllerGen). Allergy 2008; 63 Suppl 86:8-160.

15. Long A, McFadden C, DeVine D, Chew P, Kupelnick B, Lau J. Management of allergic and nonallergic rhinitis. Evid Rep Technol Assess (Summ) 2002;(54):1-6.

16. Bauchau V, Durham SR. Prevalence and rate of diagnosis of allergic rhinitis in Europe. Eur Respir J 2004;24:758-64.

17. Bachert C, van Cauwenberge P, Olbrecht J, van Schoor J. Prevalence, classification and perception of allergic and nonallergic rhinitis in Belgium. Allergy 2006;61:693-8.

18. Nathan RA, Meltzer EO, Derebery J, Campbell UB, Stang PE, Corrao MA, Allen G, Stanford R. The prevalence of nasal symptoms attributed to allergies in the United States: findings from the burden of rhinitis in an America survey. Allergy Asthma Proc 2008;29:600-8.

19. Choi BC, McQueen DV, Puska P, Douglas KA, Ackland M, Campostrini S, Barceló A, Stachenko S, Mokdad AH, Granero R, Corber SJ, Valleron AJ, Skinner HA, Potemkina R, Lindner MC, Zakus D, de Salazar LM, Pak AW, Ansari Z, Zevallos JC, Gonzalez M, Flahault A, Torres RE. Enhancing global capacity in the surveillance, prevention, and control of chronic diseases: seven themes to consider and build upon. J Epidemiol Community Health 2008;62:391-7. 
20. Zhang L, Han D, Huang D, Wu Y, Dong Z, Xu G, Kong W, Bachert C. Prevalence of self-reported allergic rhinitis in eleven major cities in china. Int Arch Allergy Immunol 2009;149:47-57.

21. Bachert C, Jorissen M, Bertrand B, Khaltaev N, Bousquet J. Allergic Rhinitis and its impact on asthma update (ARIA 2008). The Belgian perspective. B-ENT 2008;4:253-7.

22. Shen J, Ke X, Hong S, Zeng Q, Liang C, Li T, Tang A. Epidemiological features of allergic rhinitis in four major cities in Western China. J Huazhong Univ Sci Technolog Med Sci 2011;31:433-40.

23. Wang ZH, Lin WS, Li SY, Zhao SC, Wang L, Yang ZG, Chen J, Zhang $\mathrm{ZF}, \mathrm{Yu}$ JZ. Analysis of the correlation of prevalence in allergic rhinitis and other allergic diseases. Zhonghua Er Bi Yan Hou Tou Jing Wai Ke Za Zhi 2012;47:379-82.

24. Wang ZH, Lin WS, Li SY, Zhao SC, Wang L, Yang ZG, Chen J, Zhang $\mathrm{ZF}$, Yu JZ. Research on prevalence and related factors in allergic rhinitis. Zhonghua Er Bi Yan Hou Tou Jing Wai Ke Za Zhi 2011;46: 225-31.

25. Wang ZH, Lin WS, Wang L, Wang XQ, Chen J, Zhang ZF, Yu JZ. Distribution of allergens in patients with allergic rhinitis in Hebei villagers and Tianjin citizens. Zhonghua Er Bi Yan Hou Tou Jing Wai Ke Za Zhi 2012;47:809-12.

26. Weiland SK, Björkstén B, Brunekreef B, Cookson WO, von Mutius E, Strachan DP; International Study of Asthma and Allergies in Childhood Phase II Study Group. Phase II of the International Study of Asthma and Allergies in Childhood (ISAAC II): rationale and methods. Eur Respir J 2004;24:406-12.

27. Ellwood P, Asher MI, Beasley R, Clayton TO, Stewart AW; ISAAC Steering Committee. The international study of asthma and allergies in childhood (ISAAC): phase three rationale and methods. Int J Tuberc Lung Dis 2005;9:10-6.

28. Asher MI, Montefort S, Björkstén B, Lai CK, Strachan DP, Weiland SK, Williams H; ISAAC Phase Three Study Group. Worldwide time trends in the prevalence of symptoms of asthma, allergic rhinoconjunctivitis, and eczema in childhood: ISAAC Phases One and Three repeat multicountry cross-sectional surveys. Lancet 2006;368:733-43.

29. Leung R, Ho P, Lam CW, Lai CK. Sensitization to inhaled allergens as a risk factor for asthma and allergic diseases in Chinese population. J Allergy Clin Immunol 1997;99:594-9.

30. Zhao T, Wang HJ, Chen Y, Xiao M, Duo L, Liu G, Lau Y, Karlberg J. Prevalence of childhood asthma, allergic rhinitis and eczema in Urumqi and Beijing. J Paediatr Child Health 2000;36:128-33.

31. Lau YL, Karlberg J. Prevalence and risk factors of childhood asthma, rhinitis and eczema in Hong Kong. J Paediatr Child Health 1998;34: 47-52.

32. Wong GW, Hui DS, Chan HH, Fok TF, Leung R, Zhong NS, Chen YZ, Lai CK. Prevalence of respiratory and atopic disorders in Chinese schoolchildren. Clin Exp Allergy 2001;31:1225-31.

33. Ma Y, Zhao J, Han ZR, Chen Y, Leung TF, Wong GW. Very low prevalence of asthma and allergies in schoolchildren from rural Beijing, China. Pediatr Pulmonol 2009;44:793-9.

34. Chiang LC, Chen YH, Hsueh KC, Huang JL. Prevalence and severity of symptoms of asthma, allergic rhinitis, and eczema in 10- to 15-year-old schoolchildren in central Taiwan. Asian Pac J Allergy Immunol 2007;25:1-5.

35. Kao CC, Huang JL, Ou LS, See LC. The prevalence, severity and seasonal variations of asthma, rhinitis and eczema in Taiwanese schoolchildren. Pediatr Allergy Immunol 2005;16:408-15.

36. Han Y, Zhang H. Epidemiological investigation of allergic rhinitis in the primary school students in grade three of Shihezi city. Lin
Chung Er Bi Yan Hou Tou Jing Wai Ke Za Zhi 2009;23:1074-8.

37. Zhang YM, Zhang J, Liu SL, Zhang X, Yang SN, Gao J, Zhao J, Chen H, Chen XX, Sun FX, Shen L, Wang DY. Prevalence and associated risk factors of allergic rhinitis in preschool children in Beijing. Laryngoscope 2013;123:28-35.

38. Kong WJ, Chen JJ, Zheng ZY, Shi QM, Zhou Y. Prevalence of allergic rhinitis in 3-6-year-old children in Wuhan of China. Clin Exp Allergy 2009;39:869-74.

39. Li S, Yang Q, Qiong D, Gao C. Epidemiological investigation on relativity of allergic rhinitis and recurring respiratory infection of preschool children in Shenzhen. Lin Chung Er Bi Yan Hou Tou Jing Wai Ke Za Zhi 2010;24:1060-1.

40. Li F, Zhou Y, Li S, Jiang F, Jin X, Yan C, Tian Y, Zhang Y, Tong S, Shen $\mathrm{X}$. Prevalence and risk factors of childhood allergic diseases in eight metropolitan cities in China: a multicenter study. BMC Public Health 2011;11:437.

41. Zhao J, Bai J, Shen K, Xiang L, Huang S, Chen A, Huang Y, Wang J, Ye R. Self-reported prevalence of childhood allergic diseases in three cities of China: a multicenter study. BMC Public Health 2010; 10:551.

42. Rhodes HL, Sporik R, Thomas P, Holgate ST, Cogswell JJ. Early life risk factors for adult asthma: a birth cohort study of subjects at risk. J Allergy Clin Immunol 2001;108:720-5.

43. Lau S, Nickel R, Niggemann B, Grüber C, Sommerfeld C, Illi S, Kulig M, Forster J, Wahn U, Groeger M, Zepp F, Kamin W, Bieber I, Tacke U, Wahn V, Bauer CP, Bergmann R, von Mutius E; MAS Group. The development of childhood asthma: lessons from the German Multicentre Allergy Study (MAS). Paediatr Respir Rev 2002;3:265-72. 44. Grossman J. One airway, one disease. Chest 1997;111:11S-16S.

45. Yin J, Yue FM, Wang LL, He HJ, Xu T, Zhang HY, Li H, Wen LP, Sun JL, Gu JQ, Han SM, Ye ST. The clinical study of the relationship between allergic rhinitis and allergic asthma in the patients with autumnal pollinosis. Zhonghua Yi Xue Za Zhi 2005;85:1683-7.

46. Verlato G, Corsico A, Villani S, Cerveri I, Migliore E, Accordini S, Carolei A, Piccioni P, Bugiani M, Lo Cascio V, Marinoni A, Poli A, de Marco R. Is the prevalence of adult asthma and allergic rhinitis still increasing? Results of an Italian study. J Allergy Clin Immunol 2003;111:1232-8.

47. Bråbäck L, Hjern A, Rasmussen F. Trends in asthma, allergic rhinitis and eczema among Swedish conscripts from farming and nonfarming environments. A nationwide study over three decades. Clin Exp Allergy 2004;34:38-43.

48. Simpson CR, Newton J, Hippisley-Cox J, Sheikh A. Incidence and prevalence of multiple allergic disorders recorded in a national primary care database. J R Soc Med 2008;101:558-63.

49. Bjerg A, Ekerljung L, Middelveld R, Dahlén SE, Forsberg B, Franklin K, Larsson K, Lötvall J, Olafsdóttir IS, Torén K, Lundbäck B, Janson C. Increased prevalence of symptoms of rhinitis but not of asthma between 1990 and 2008 in Swedish adults: comparisons of the ECRHS and GA(2)LEN surveys. PLoS One 2011;6:e16082.

50. de Marco R, Cappa V, Accordini S, Rava M, Antonicelli L, Bortolami O, Braggion M, Bugiani M, Casali L, Cazzoletti L, Cerveri I, Fois AG, Girardi P, Locatelli F, Marcon A, Marinoni A, Panico MG, Pirina P, Villani S, Zanolin ME, Verlato G; GEIRD Study Group. Trends in the prevalence of asthma and allergic rhinitis in Italy between 1991 and 2010. Eur Respir J 2012;39:883-92.

51. Wang HY, Zheng JP, Zhong NS. Time trends in the prevalence of asthma and allergic diseases over 7 years among adolescents in Guangzhou city. Zhonghua Yi Xue Za Zhi 2006;86:1014-20. 
52. Liao PF, Sun HL, Lu KH, Lue KH. Prevalence of childhood allergic diseases in central Taiwan over the past 15 years. Pediatr Neonatol 2009;50:18-25.

53. Yang Y, Zhao Y, Wang CS, Wang XD, Zhang L. Prevalence of sensitization to aeroallergens in 10030 patients with allergic rhinitis. Zhonghua Er Bi Yan Hou Tou Jing Wai Ke Za Zhi 2011;46:914-20.

54. Du J, Ma X, Wang J, Deng J. Investigation on the antigens responsible for allergic rhinitis occurred in the population of Kunshan district. Lin Chung Er Bi Yan Hou Tou Jing Wai Ke Za Zhi 2012;26:370-1.

55. Ding J, Zhang J, Xu F, Xu Y, Zhu H. Analyzing of the inhaled allergens profiles of 890 allergic rhinitis patients. Lin Chung Er Bi Yan Hou Tou Jing Wai Ke Za Zhi 2012;26:164-6.

56. Lü Y, Xie Z, Zhao S, Zhang H, Liu Y, Chen X, Jiang W. Prevalence of allergens for Changsha patients with allergic rhinitis. Lin Chung Er Bi Yan Hou Tou Jing Wai Ke Za Zhi 2011;25:491-4.

57. Liu J, Zhou Y, Wan J, Liu Z. Aeroallergen spectrum of patients with allergic rhinitis in Enshi area. Lin Chung Er Bi Yan Hou Tou Jing Wai Ke Za Zhi 2011;25:649-51, 655.

58. Huang F, Zhao Y, He J, Deng P, Zhou P, Zhou J, Zhao X, Yang Z. Analyzing of the inhaled allergens profiles of allergic rhinitis patients in district of Jingmen. Lin Chung Er Bi Yan Hou Tou Jing Wai Ke Za Zhi 2010;24:341-3.

59. Wang C, Zhang L, Han D, Zhou B, Zhao Y, Wang X. Prevalence of sensitization to aeroallergens in Beijing patients with allergic rhinitis. Lin Chuang Er Bi Yan Hou Ke Za Zhi 2006;20:204-7.

60. Jiang C, Li L, Tan G. Aeroallergen spectrum of 387 patients with allergic rhinitis in Changsha area. Lin Chung Er Bi Yan Hou Tou Jing Wai Ke Za Zhi 2008;22:794-7.

61. Wu W, Yalikun Y, Chen Y, Tian J, Ma L. The analysis on the allergen test of the allergic rhinitis with 1564 cases in Changji district. Lin Chung Er Bi Yan Hou Tou Jing Wai Ke Za Zhi 2011;25:789-90.

62. Celedón JC, Palmer LJ, Weiss ST, Wang B, Fang Z, Xu X. Asthma, rhinitis, and skin test reactivity to aeroallergens in families of asthmatic subjects in Anqing, China. Am J Respir Crit Care Med 2001; 163:1108-12.

63. Sun BQ, Lai XX, Gjesing B, Spangfort MD, Zhong NS. Prevalence of sensitivity to cockroach allergens and IgE cross-reactivity between cockroach and house dust mite allergens in Chinese patients with allergic rhinitis and asthma. Chin Med J (Engl) 2010;123:3540-4.

64. Li J, Huang Y, Lin X, Zhao D, Tan G, Wu J, Zhao C, Zhao J, Spangfort MD, Zhong N; China Alliance of Research on Respiratory Allergic Disease (CARRAD). Influence of degree of specific allergic sensitivity on severity of rhinitis and asthma in Chinese allergic patients. Respir Res 2011;12:95.

65. Dai WL, Zhang J, Pan YS, Chen M, Zhang YM. Clinical analysis of allergic rhinitis in children between 1 and 3 years of age. Zhonghua Er Bi Yan Hou Tou Jing Wai Ke Za Zhi 2011;46:921-7.

66. Zhong Z, Wang F, Wang T, Li L, Tan G. Aeroallergen spectrum of patients with child allergic rhinitis in Changsha area of China. Lin Chung Er Bi Yan Hou Tou Jing Wai Ke Za Zhi 2011;25:774-6.
67. Liu GH, Zhu RF, Zhang W, Li WJ, Wang ZX, Chen H. Survey of airborne pollen in Hubei province of China. Chin Med Sci J 2008;23: 212-7.

68. Ouyang YH, Zhang DS, Fan EZ, Li Y, Zhang L. Correlation between symptoms of pollen allergic rhinitis and pollen grain spreading in summer and autumn. Zhonghua Er Bi Yan Hou Tou Jing Wai Ke Za Zhi 2012;47:623-7.

69. Pénard-Morand C, Charpin D, Raherison C, Kopferschmitt C, Caillaud D, Lavaud F, Annesi-Maesano I. Long-term exposure to background air pollution related to respiratory and allergic health in schoolchildren. Clin Exp Allergy 2005;35:1279-87.

70. Bhattacharyya N. Air quality influences the prevalence of hay fever and sinusitis. Laryngoscope 2009;119:429-33.

71. Mösges R, Klimek L. Today's allergic rhinitis patients are different: new factors that may play a role. Allergy 2007;62:969-75.

72. Hsu SP, Lin KN, Tan CT, Lee FP, Huang HM. Prenatal risk factors and occurrence of allergic rhinitis among elementary school children in an urban city. Int J Pediatr Otorhinolaryngol 2009;73:807-10.

73. Kim H, Bernstein JA. Air pollution and allergic disease. Curr Allergy Asthma Rep 2009;9:128-33.

74. Krämer U, Koch T, Ranft U, Ring J, Behrendt H. Traffic-related air pollution is associated with atopy in children living in urban areas. Epidemiology 2000;11:64-70.

75. Li Y, Wang W, Wang J, Zhang X, Lin W, Yang Y. Impact of air pollution control measures and weather conditions on asthma during the 2008 Summer Olympic Games in Beijing. Int J Biometeorol 2011;55:547-54.

76. Zhang F, Wang W, Lv J, Krafft T, Xu J. Time-series studies on air pollution and daily outpatient visits for allergic rhinitis in Beijing, China. Sci Total Environ 2011;409:2486-92.

77. Hwang BF, Jaakkola JJ, Lee YL, Lin YC, Guo YL. Relation between air pollution and allergic rhinitis in Taiwanese schoolchildren. Respir Res 2006;7:23.

78. Lee YL, Shaw CK, Su HJ, Lai JS, Ko YC, Huang SL, Sung FC, Guo YL. Climate, traffic-related air pollutants and allergic rhinitis prevalence in middle-school children in Taiwan. Eur Respir J 2003;21:964-70.

79. Yu JH, Lue KH, Lu KH, Sun HL, Lin YH, Chou MC. The relationship of air pollution to the prevalence of allergic diseases in Taichung and Chu-Shan in 2002. J Microbiol Immunol Infect 2005;38:123-6.

80. Chang CC, Lee IM, Tsai SS, Yang CY. Correlation of Asian dust storm events with daily clinic visits for allergic rhinitis in Taipei, Taiwan. J Toxicol Environ Health A 2006;69:229-35.

81. Gao RL, Ding J, Zang YW, Yan S, Liu TT, Liu ZG, Gong XG, Zhou XB, Li N, Zhang NK. Prevalence of allergic rhinitis and survey analysis of health service needs and utilization in Qingdao area. Zhonghua Er Bi Yan Hou Tou Jing Wai Ke Za Zhi 2012;47:633-7.

82. Zhang L, Wei JM, Han DM. Current state of diagnosis and treatment of allergic rhinitis in China. Zhonghua Er Bi Yan Hou Tou Jing Wai Ke Za Zhi 2010;45:420-3. 